\title{
Analytical model and experimental validation of the heat transfer and the induced flow in a PV cooling duct in environmental conditions
}

\author{
R. Mazón ${ }^{1, *}$, A.S. Káiser ${ }^{1, *}$, B. Zamora ${ }^{1}$, J.R. García ${ }^{1}$, F. Vera ${ }^{1}$ \\ ${ }^{1}$ Thermal and Fluid Engineering Department, Technical University of Cartagena, Doctor Fleming, s/n 30202, \\ Cartagena, Murcia, Spain \\ *Tel:3496832594rmh@alu.upct.es \\ *Tel: 34 968325984, antonio.kaiser@upct.es
}

\begin{abstract}
This paper describes a model to account for the heat transfer and the convective flow induced in the interior of a channel inclined $35^{\circ}$ with respect to the horizontal, formed by a photovoltaic panel and an adiabatic plate. The model developed is validated experimentally by measurements made on an experimental prototype. The solar installation consists of two photovoltaic panels integrated with air ducts on the top of a building in southern Spain. The objective is to determine the temperature reached by the photovoltaic module in this configuration. This model considers the processes of heat transfer by radiation and convection on the outside for several atmospheric conditions (wind speed, ambient temperature and incident radiation) and for different geometrical and physical characteristics of the PV cooling duct (plate area, module performance, emission and absorption coefficients). Good agreement has been obtained between the experimental data and the results of this model.
\end{abstract}

Keywords: PV cooling duct, solar experimental facilities, efficiency solar panels.

\section{Introduction}

The performance of PV implemented on the top of a building depends on the panel temperature in addition to other factors such as insulation and shading. High panel temperatures resulting from overheating could occur due to low wind cooling effect compared with free standing installation. One cost effective method to regulate the temperature of rooftop integrated photovoltaic (PV) panels is to provide an open air channel underneath the panel.

To determine the equilibrium temperature of the modules, for different atmospheric conditions, it is necessary to solve simultaneously the heat transfer processes between the PV modules and the exterior and interior of the air duct, and the convective flow induced by buoyancy effects and by the suction of the external wind. Various studies have been carried out to assess the performance of roof-mounted solar (thermal or photovoltaic) panels. Some authors centered their research on the effect of the air gap between a solar panel and a roof [1], others suggested that a reasonable air gap for solar thermal collectors would be between 0.1 and $0.14 \mathrm{~m} \mathrm{[2]} \mathrm{and} \mathrm{also} \mathrm{derived} \mathrm{expressions} \mathrm{for} \mathrm{the} \mathrm{mass} \mathrm{flow} \mathrm{rate,} \mathrm{velocity} \mathrm{and}$ temperature rise in the air gap behind solar cells considering the effect of the geometry (aspect ratio of the channel) and location of these solar cells [3]. Other authors presented procedures of the heat transfer and the convective flow through a PV cooling duct to determine the equilibrium temperature of the module [4].

This work initially raises the set of equations that characterize the thermal and fluid dynamic process in the air gap behind the solar cells. Subsequently a selection of the characteristic values is made of the input variables in this model, based on the existing literature and the available data on the experimental prototype presented in this work. Once estimated the solution of the equations system is presented, with particular emphasis on how the temperature of the module changes as the incident radiation and the external wind change. To 
solve this system of equations the ESS software has been used. The model is validated with experimental measurements obtained on the configuration under study.

\section{Experimental facility}

The solar installation consists of two photovoltaic panels arranged as shown in figure 1, first panel (panel A) is used as reference panel. Panel temperature at different points, voltage, and current are measured to understand the panel behavior under normal operating conditions and to compare with another one (panel B) which is modified to test different ducts with different cross sections. In this second panel, surface temperature at different points, voltage, and current are also measured jointly with the air temperature and air flow rate. In the solar panels, temperatures are measured with RTD, and duct air speed with hot film anemometers [5].

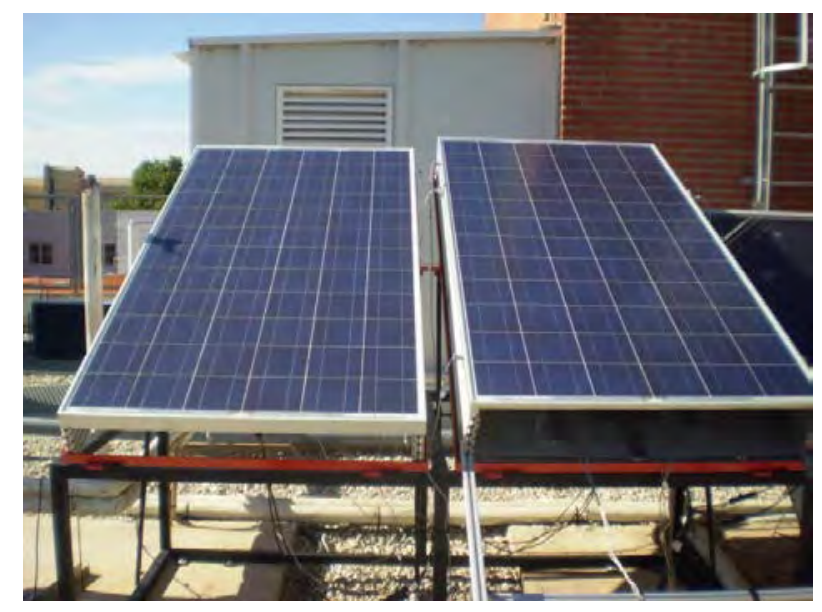

Fig. 1. Solar panels. Left panel (A), right panel (B).

The horizontal components of solar radiation are measured by two pyranometers. Other environmental conditions such as temperature, pressure, humidity and wind speed are measured with a meteorological station place on our laboratory roof just beside the experimental facility. All data is registered and recorded by means of a data logger.

\section{Analytical model}

\subsection{The incoming heat flow to the channel}

As mentioned, the procedure requires the simultaneous solution of two heat transfer equations. On one hand it has to solve the external equation, which represents the transfer of heat to the surroundings from the top face of the module and on the other hand the internal equation, which represents the transfer of heat into the duct. The module temperature links up both equations. In general, there may be a temperature difference between the two faces of the photovoltaic module, which is given by the expression [6]

$$
T_{o}-T_{m}=\frac{E}{E_{o}} \Delta T
$$

where $T_{o}$ is the cell temperature inside the module, $T_{m}$ the lower face temperature, $E$ is the irradiance measured, $E_{o}$ is reference solar irradiance on the module $\left(1000 \mathrm{~W} / \mathrm{m}^{2}\right)$ and $\Delta T$ the temperature gap between cell and back surface measured with $E_{o}=1000 \mathrm{~W} / \mathrm{m}^{2}$, whose value 
depends on the module rear ventilation (in this case is usually $2-3^{\circ} \mathrm{C}$ ). In the approach of our model we consider in the first instance that there is no temperature difference between the two sides of the module.

The external equation requires the following energy balance. At any point of the module, the heat flux that goes into the channel is given by the difference between the absorbed solar radiation minus the electrical output and losses to the surroundings. These losses are due on one hand, to the reflected and emitted radiation, and on the other hand to the external convection:

$$
q=\left(\alpha_{s}-\eta\right) \cdot q_{s}-U_{a}\left(T_{o}-T_{a}\right)
$$

being $\alpha_{s}$ the absorption coefficient, $\eta$ the electrical efficiency, $U_{a}$ the external loss coefficient and $T_{a}$ the ambient temperature, and $q_{s}$ the solar flux or irradiance. As guide values for electrical efficiency and the absorption coefficient we've considered $\alpha_{s}=0,85$ and $\eta=0,12$ [7]. Other authors suggest a value of $\alpha \mathrm{s}-\eta=0.8$ and regarding the external loss coefficient ( $U_{a}$ ), they also propose $U_{a}=h_{r e}+h_{c e}[8]$ where

$$
\begin{gathered}
h_{r e}=4 \varepsilon \sigma\left[\frac{T_{o}+T_{a}}{2}\right]^{3} \\
h_{c e}=1,3\left(T_{o}-T_{a}\right)^{1 / 3}+4 \cdot V_{10}
\end{gathered}
$$

with $h_{r e}$ as the radiation loss coefficient (where we assume that the surroundings are a cavity of adjacent air temperature and that the panel has an emittance of $\varepsilon$ ), $h_{c e}$ is the external convection coefficient (it takes into account the existing exterior wind and it has been adjusted for the location of our facility studied considering the results of Palyvos [9]) and $V_{10}$ is the external wind speed at the height of 10 meters. It is obvious that in general the heat flow $q$ is not uniform over the surface of the module. However, as $U_{a}$, is usually taken as uniform, so the heat flow will be also in our model over the PV module.

The formulation of the equation of heat transfer inside the duct initially requires determining the buoyancy-induced flow and wind effects inside the channel when the photovoltaic module is subjected to a uniform solar radiation. In this process, the output variables are the wall temperatures, $T_{o}$ (module temperature) and $T_{b}$ (temperature of adiabatic wall) and its variation along the panels. The process requires an iterative calculation, given the existing degree of coupling.

\subsection{The mass flow rate induced inside the channel}

According to the investigations of Brinkworth [8], considering a constant heat flow in the module, determining the flow induced in the interior, is decoupled from the temperature of the channel walls, and can therefore be obtained separately. Therefore, we will raise the problem as decoupled and initially we will solve the induced mass flow (and hence the average speed) and then, based on this result, the heat transfer and temperature of the plates. To obtain the induced mass flow inside the channel, it is necessary to consider a balance of global forces on the air mass inside the same channel. The flow inside the duct is the result of driving forces (buoyancy-induced by natural convection and wind suction) and resistant forces (friction and 
other hydraulic losses in the inputs and outputs). Below, this paper describes each of these forces.

\subsection{Driving forces}

The drop in pressure to conduct the air through the duct is the sum of buoyancy and wind effect [10]:

$$
\Delta p_{d}=\Delta p_{b}+\Delta p_{w}
$$

For flows that meet certain conditions, Boussinesq suggested that variations in fluid density can be neglected except in the gravitational term of the equation of conservation of momentum in the vertical direction, in which density appears multiplied by the gravity acceleration $(g)$. This approach assumes that other fluid properties such as dynamic viscosity, conductivity and specific heat are constant. Supposing that the compressibility effects are small, so that density variations are due solely to the temperature changes, we can use the Boussinesq approximation, if the temperature variations in the fluid are small. Considering the Boussinesq's hypothesis the buoyancy term can be expressed as the difference between the base and the end point of the fluid column with a height of $L \sin \theta$ :

$$
\Delta p_{b}=\rho g \beta\left(T_{\text {media }}-T_{i}\right) L \sin \theta=\rho g \beta S\left(T_{e}-T_{i}\right) L \sin \theta
$$

where $S\left(T_{e}-T_{i}\right)$ is the average increases of the temperature reached in the duct. The value of $S$ depends on the temperature profile along the duct. For a linear increase in temperature, its value is about 0.5 . This value is what we consider in this study as a starting point. On the other hand, the value of ( $T e-\mathrm{Ti}$ ) can be expressed in terms of incoming heat as

$$
q A=\dot{m} C_{p}\left(T_{e}-T_{i}\right)
$$

If we solve eq. (7) for $\left(T_{e}-T_{i}\right)$, expressing the mass flow-rate, $\dot{m}$, in terms of the density,

$$
\left(T_{e}-T_{i}\right)=\frac{q L}{\rho U H C_{p}}
$$

and substituting in equation (6)

$$
\Delta p_{b}=\frac{g \beta S q L^{2} \sin \theta}{U H C_{p}}
$$

where $\beta$ is the coefficient of thermal expansion, $L$ the channel length, $U$ the average velocity of induced flow, $C_{p}$ the specific heat of air and $H$ the depth of the channel. The channel width is taken to be $1 \mathrm{~m}$.

As already mentioned, the external wind exerts a suction force on the channel so the mass flow increases. The pressure difference induced by wind can be represented by

$$
\Delta p_{w}=C_{p i}\left(\frac{\rho V_{w i}^{2}}{2}\right)-C_{p e}\left(\frac{\rho V_{w e}^{2}}{2}\right)
$$


being $C_{p i}$ and $C_{p e}$ the wind pressure coefficients at the entrance and exit of the duct, and $V_{w i}$ and $V_{w e}$ are the values of wind speed at the inlet and outlet of the duct. According to Brinkworth [8], assuming that the position of the duct is such that the wind causes an increase in mass flow, we can estimate the combined effect of wind on the channel as:

$$
\Delta p_{w}=(+0.5) \frac{\rho V_{10}^{2}}{2}
$$

with $V_{10}$ the wind speed at the height of 10 meters above the channel (the coefficient of +0.5 is included in eq.11 to adjust experimentally the effect of the surroundings).

\subsection{Resistant forces}

The flow resistance expressed in terms of pressure differences $\left(\Delta p_{r}\right)$ is given by the sum of the pressure drop due to friction $\left(\Delta p_{f}\right)$ and other hydraulic losses $\left(\Delta p_{h}\right)$. Friction losses can be expressed by the Darcy's equation

$$
\Delta p_{f}=f(L / D) \frac{\rho U^{2}}{2}
$$

where $f$ is the friction factor in a duct of length $L$ and the hydraulic diameter of the cross section is $D=2 H$, due to the width is $1 \mathrm{~m}$ and $\mathrm{H}<<1$. In general, the factor $f$ is a function of the aspect ratio $(L / D)$ and of the Reynold number $\left(R e_{D}=U D / v\right)$. In the case of a channel the following expression is proposed:

$$
f=f_{o}+\frac{f_{1}}{L / D}+\frac{f_{2}}{\operatorname{Re}^{n}}
$$

With $\mathrm{n} \approx 1, \mathrm{f}_{0} \approx 0, \mathrm{f}_{1} \approx 1$ and $\mathrm{f}_{2} \approx 64$. Given these values, the expression $f(L / D)$ approaches to 1. On the other hand, the pressure drop due to hydraulic losses can be expressed in terms of coefficients $\mathrm{K}_{\mathrm{h}}$ of the dynamic pressure in the form

$$
\Delta p_{h}=\left(\sum k_{h}\right) \frac{\rho U^{2}}{2}
$$

When there is a heat flow asymmetry on both walls in the channel. We may assume $\sum k_{h} \approx 1.5$ [10]. If we propose the balance of forces, the equation obtained is:

$$
\frac{2 g q S L^{2} \sin \theta}{T_{a} \rho H C_{p}}=\left(f \frac{L}{D}+\sum k_{h}\right) U^{3}-\left(\frac{2 \Delta p_{w}}{\rho}\right) U
$$

which is a cubic function of the average velocity induced in the channel.

$$
A \cdot U^{3}+B \cdot U^{2}+C \cdot U+X=0
$$

With $A=1+\sum k_{h}, \quad B=0, C=-V_{10}^{2}$ and $X=-2 g q S L^{2} \sin \theta / T_{a} H \rho C_{p}$. 


\subsection{The heat transfer inside the duct}

The incoming heat flow $q$ is transferred to the air through the convection at the two walls of the channel and may be expressed by $q=q_{0}+q_{b}$. The first term, $q_{o}$, regards to the heat flow for convection of the PV module to channel air. This is given by

$$
q_{0}=\left(T_{0}-T_{m}\right) \cdot h_{0}
$$

where $h_{o}$ the convection coefficient of this panel, $T_{o}$ temperature of the panel at a distance of the channel input in longitudinal direction and $T_{m}$ the average temperature of the air-flow at this point. The second term, $q_{b}$, represent the heat flow for convection from the adiabatic module. This heat came from the radiation heat flow issued over this panel for the PV module. It can be represented with the next equation.

$$
q_{b}=\left(T_{b}-T_{m}\right) \cdot h_{b}
$$

This radiation heat flow can be well represented for an interchange direct equation that considers local temperature of the two facing surfaces, with a heat transferred coefficient linearized $h_{r}$, described by [8],

$$
h_{r}=4 \cdot \varepsilon_{e f f} \cdot \sigma\left(\frac{T_{0}+T_{b}}{2}\right)^{3}
$$

In this case, we assume that the plate facing the module is adiabatic and that the energy that it receives by the radiation is entirely transferred for convection to the flow. So, the average air temperature in the duct at a distance $x$ from the input is given by

$$
\left(T_{m}-T_{b}\right)=\frac{q x}{\rho_{a} C_{p} U H}
$$

From the above equations, you can get the temperatures of the two walls thanks to the expressions (23) and (24). The convection coefficients $h_{o}$ and $h_{b}$ can be obtained from the following expressions

$$
\begin{gathered}
h_{0} \cdot(1-n \hat{\theta})=h_{c} \\
h_{b} \cdot(1-\hat{\theta} / n)=h_{c}
\end{gathered}
$$

where $h_{c}$ is the coefficient of convective heat transfer on the hot wall of a channel in where the other wall is adiabatic and we assume there is not heat flow by radiation. $\hat{\boldsymbol{\theta}}$ is the influence coefficient which depends on the shape of the temperature profile of the plate that the duct is only heated on one side. The variable $\mathrm{n}$ is the ratio of heat flux between two walls

$$
n=\frac{q_{b}}{q_{0}}=\frac{h_{r}\left(T_{0}-T_{b}\right)}{h_{0}\left(T_{0}-T_{m}\right)}
$$


To close the system, we need information about $h_{c}$ (or of $\mathrm{Nu}_{\mathrm{c}}$ ) and $\hat{\theta}$. For turbulent flow in a channel with heat flow in a plate and the other being adiabatic, the following expression is referenced, where $\mathrm{Re}_{\mathrm{D}}$ is the Reynolds number based on the hydraulic diameter:

$$
h_{c}=0,0186 \operatorname{Re}_{D}^{0,787} k / D
$$

With regards to the value of $\hat{\theta}$, we have estimated it, correlating the presented values for the case of $\operatorname{Re}_{D}=10,000$ according [11]

$$
\hat{\theta}=0,0452 \ln (L / D)+0,0178
$$

In spite of these values assumed by $h c$ (or by $N u_{c}$ ) and of $\hat{\theta}$, there is no criteria in bibliography to determine them. Therefore, a future task is to numerically determine its values for different conditions of heat flow between both walls and different angles of incidence.

\section{Experimental validation of the model and results}

The equation model proposed to resolve the heat transfer and the induced flow has been solved by means of the EES software used, for different real cases which have been measured experimentally in our solar facility. The following starting parameters have been considered: $C p=1007 \mathrm{~J} / \mathrm{kg} \mathrm{K}, D_{h}=0.27 \mathrm{~m}, \varepsilon=0.88, \quad \varepsilon_{\text {eff }}=0.3, g=9.8 \mathrm{~m} / \mathrm{s}^{2}, \eta=0.1353, H=0.165$ $\mathrm{m}, \alpha_{s}=0.85, K_{h}=1.5, L=1.956 \mathrm{~m}, \sigma=5.670 \times 10^{-8} \mathrm{~W} / \mathrm{m}^{2} \mathrm{~K}^{4}, \mu_{a}=0.00001834 \mathrm{~kg} / \mathrm{ms}, V_{w}$ $=1,86 \mathrm{~m} / \mathrm{s}, R e_{D h}=17,351, \rho_{a}=1.197 \mathrm{~kg} / \mathrm{m}^{3}, S=0.5$ y $\theta_{p}=35^{\circ}$.

Figure 2 compares the experimental data of the temperatures of the module $\left(T_{o}\right)$ and the adiabatic wall $\left(T_{b}\right)$ as a function of the irradiance, with those obtained by the analytical model. Taking into account that the experimental data has been obtained varying the atmospheric temperature (between 298 and $303 \mathrm{~K}$ ), the results of the analytical model have been presented for different atmospheric temperature conditions. Experimental correlations for module and adiabatic wall temperature have been also printed in the Fig. 2. On the other hand, it has been also studied experimental and analytically the effect of the air gap between the module and the adiabatic wall. It has been tested that its effect is not very important in the performance of the module, once a minimum value of the aspect ratio has been reached $(H / L \approx 0.1)$.

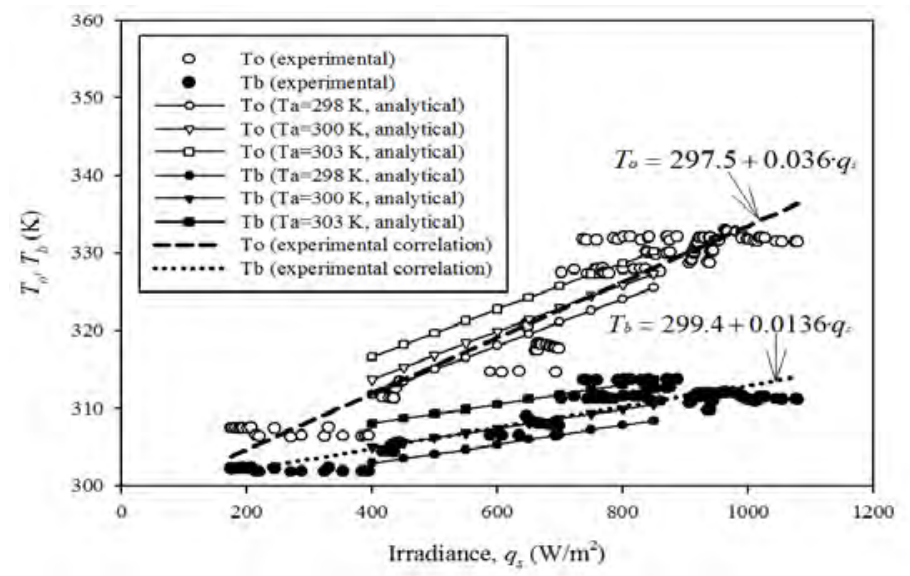


Fig.2. Experimental validation of the analytical model for different atmospheric temperatures and the experimental correlation of the photovoltaic plate temperatures.

\section{Conclusions}

A solar installation consisted of a photovoltaic panel and adiabatic plate in an inclined channel configuration has been experimentally tested. Experimental correlations have been proposed for the temperature of the module and the temperature of the adiabatic plate as a function of the irradiance, for an average wind velocity of $2 \mathrm{~m} / \mathrm{s}$ and an average atmospheric temperature of roughly $300 \mathrm{~K}$.

An analytical model of this configuration to study the heat transfer and the convective flow induced in it has been carried out and solved by EES software, considering some previous models of the bibliography and particular aspects of this experimental facility. The experimental measurements have been used to validate this analytical model. It has been tested that the effect of the air gap between the module and the adiabatic wall is not very important in the performance of the module, once a minimum value of the aspect ratio has been reached $(H / L \approx 0.1)$.

\section{Acknowledgements}

The authors thank the local association Fundacion Seneca for its cooporation in this study, which has been supported by the company Apia XXI, so we'd like to show our gratitude to them.

\section{References}

[1] J. Khedari, J. Hirunlabh, T. Bunnag, Experimental study of a roof solar collector towards the natural ventilation of new habitations. Energy and Buildings 26, 1997, pp. 159-164.

[2] J. Hirunlabh, S. Wachirapuwadon, N. Pratinthong, J. Khedari, New configuration of a roof solar collector maximizing natural ventilation. Building Env. 36, 2001, pp. 383-391.

[3] M. Sandberg, B. Moshfegh, Buoyancy-induced air flow in photovoltaic facades. Effect of geometry of the air gap and location of solar cell modules. B. Env. 37, 2002, pp. 211-218

[4] B.J. Brinkworth, R.H. Marshall, Z Ibarah, A validated model of naturally ventilated PV cladding. Solar Energy 69 (1), 2000, pp. 67-81.

[5] R. Mazón, JR García, F Vera, A S. Kaiser, B Zamora, Development of an installation to reduce the temperature of photovoltaic modules and improve their efficiency, ICREPQ'10, 2010.

[6] D.L. King, W.E. Boyson, J.A. Kratochvil, Photovoltaic array performance model, Sandia National Laboratories, 87185-0752, 2004.

[7] F. Sarhaddi, S. Farahata, H. Ajama, A. Behzadmehra and M. Mahdavi Adelia, An improved thermal and electrical model for a solar photovoltaic thermal (PV/T) air collector, Applied Energy 87, 2010, pp. 2328-2339.

[8] B.J. Brinkworth, M. Sandberg, Design procedure for cooling ducts to minimize efficiency loss due to temperature rise in PV arrays, Solar Energy 80, 2006, pp. 89-103.

[9] J.A. Palyvos, A survey of wind convection coefficient correlations for building envelope energy systems'modeling, Applied Thermal Engineering 28, 2008, pp. 801-808.

[10] B.J. Brinkworth, Estimation of flow and heat transfer for the design of PV cooling ducts, Solar Energy 69, 2000, pp. 413-420. 
[11] B.J. Brinkworth, Coupling of convective and radiative heat transfer in PV cooling ducts, ASME 124, 2002, pp. 250-255. 